\title{
Thermodynamic analysis of systems anaerobic fermentation
}

\section{Oleksandr Shevchenko, Anatolii Sokolenko, Kostiantyn Vasylkivskii, Oleg Stepanets}

\author{
National University of Food Technologies, Kyiv, Ukraine
}

\section{Keywords:}

Mass transfer

Energy transfer

Bacteria

Anaerobic

Brood

Entropy

Thermodynamics

Article history:

Received 16.05.2017

Received in revised

form 11.08.2017

Accepted 05.09.2017

\section{Corresponding}

author:

Oleksandr Shevchenko

E-mail:

tmipt@ukr.net

DOI: $10.24263 / 2304-$

974X-2017-6-3-15

\section{Abstract}

Introduction. The article deals with the use of peculiarities of processes of micro-biological and thermodynamic transformations in digestible media, which are combined by the principles of minimizing of energy potentials and targeting to the most probable states.

Materials and methods. The research methods are based on the study of the peculiarities of material and energy transformations in the wadding cycles due to entropy transformations in biological systems.

Results and discussion. The course of technologies of anaerobic and aerobic fermentation is a local component of the general carbon cycle, in which there are entropy transformations and losses in the form of thermal energy. The self-sufficiency and irreversibility of the processes of preparation of sugarcontaining environments and the fermentation process itself is a reflection of the laws of thermodynamics, on the basis of which the system of utilization of fermentation heat is proposed.

The technologies of anaerobic and aerobic fermentation are those that have the potential of non-traditional energy sources, the use of which is possible on the theoretical basis of heat pumps. According to R. Clausius, the course of natural processes is self-destructive and it concerns chemical and biochemical reactions. This provision relates to technical systems that supply natural self-sustaining phenomena and processes, in which, among others, the natural properties of yeast- sugar mites are realized. The thermodynamic evaluation of biological systems in the form of the characteristics of the seams of the parts of the entropy change and the energy transformations of the nutritional components with the participation of ATP and ADP and the free energy of Gibbs, shows that saturation of the culture medium with carbon dioxide creates obstacles in the path of endogenous processes of the synthesis of ethyl alcohol and carbon dioxide. The scheme of utilization of secondary energy resources of fermentation processes is proposed, taking into account that the potential of fermentation heat is almost twice the heat of distillation.

Conclusions. The amount of heat of fermentation is sufficient for the distillation process with parallel connection of these processes, and the magnitude and speed of generation of free Gibbs energy in culture media is related to the dynamics of growth of its entropy. 


\section{Introduction}

Solving the problems of energy supply of modern technologies is carried out on the ways of searching for non-traditional energy sources with the creation of new systems and simultaneously at the expense of secondary energy resources. In the latter case, the technical possibilities of recovery and regeneration of energy-material flows, including those on the basis of closed energy circuits, are created. Understanding that the entire biological world of the Earth exists in the ocean of energy always pushes humanity to seek opportunities for its redistribution. So in 1852, William Thomson Lord Kelvin proposed to use a heat sink for heating facilities on the basis of refrigerating cycles. Since then, extensive use of heat pumps in various forms began, including air conditioners of various applications $[1,2,7]$. The theoretical basis of the converters of low potential energy sources on a high potential relates to the Carnot inverse cycle and the Rankine's cycle for wet pairs in accordance with the laws of thermodynamics. Particularly useful heat pumps are in cases of their dual purpose, when using cooling and simultaneously heating different environments. One of the possible cases of such use may be the temperature stabilization of the sorted ethanol environments and the heat supply of the masonry or distillation columns. It is known that the production of one moth of sugar in anaerobic conditions is accompanied by the allocation of $169 \mathrm{~kJ}$ of thermal energy, which, in the first place, is lost in modern technologies, and, secondly, requires additional material and energy costs. In connection with this, the necessity for in-depth study of the thermodynamics of thermal processes in the collections of fermentation and distillation technologies and the creation of closed circuits is inevitable.

\section{Materials and methods}

The research methods are based on the study of the peculiarities of material and energy transformations in the wadding cycles due to entropy transformations in biological systems. The basis of the research was the well-known laws of technologies, approximating to the provisions of thermodynamics and phenomenological considerations.

\section{Result and discussion}

According to Rudolf Clausius, natural processes in their movements are self-inflating. Sometimes they are called "non-compensated" $[3,4,15]$. It is known that in steady-state equilibrium in mechanical systems, the equilibrium is equivalent to the minimum of the potential energy. It is natural to assume that the chemical processes under the self-flow flow are directed towards the reduction of the internal energy of the system. At the same time, the principle of orientation of processes to the most reliable state takes place, which corresponds to the most unordered form of particle distribution. The combination of these two principles is accompanied by heat conduction, re-creation of work into heat, diffusion processes. However, in the opposite direction, their course "by itself" without compensation is impossible. Irreversible processes are possible, but they do not have the course "by themselves". In every direct circular carrier-free process, the transformation of heat into work is offset by the simultaneous self-governing process of transferring part of the summed heat from the heat transfer to the heat-receiving device. 
Any unscrupulous process occurs only after its accompaniment by the self-governing process. The second Clausius integral has the form:

$$
\oint \delta Q / T \leq 0
$$

where the sign "equals" refers to the reverse processes, and the sign "less" - to non-inverse.

Entropy is a state function, therefore, the change in entropy for both inverse and irreversible processes will be the same. For every process $d s \geq \delta Q / T$, where $\delta \mathrm{Q}$ is the amount of perceived heat, $s=\int \delta Q / T+s_{0}$, where the integration state $s_{0}$ can not be determined within the framework of the first and second laws of thermodynamics. Of course, values $s_{0}$ are chosen arbitrarily, focusing on practical needs. All smooth processes, which proceed from the states less likely to be more probable, are irreversible and associated with an increase of entropy. The general laws of the course of processes in inanimate and biological systems are based on the first and second laws of thermodynamics, but in the first case the first section of the classical (equilibrium) thermodynamics is used, and in the second, the thermodynamics of irreversible processes (non-equilibrium) is used. Biological systems are open-ended, irreversible and nonequilibrium, and their transition from one state to another is impossible without the additional inflow of energy from the outside and the basic notion about them is the stationary state of the system. In stationary states, gradients of parameters are supported.

In accordance with the second law of thermodynamics, the change in entropy ds is greater than or equal to the absorbed system of elementary reduced heat:

$$
d s \geq \frac{\delta Q}{T}
$$

For an isolated system $(\delta \mathrm{Q}=0)$ we have $d s \geq 0$. In the inverse (equilibrium) processes, the entropy is unchanged (the sign is "equal"), in irreversible it increases to maximum (sign "more"). This corresponds to the Clausius criterion and the isolated system is directed toward achieving a finite equilibrium with maximum entropy.

Thus, the second law of thermodynamics indicates the direction of the processes in the system. Even in the case of unexpected fluctuations, which led to a decrease in entropy, the subsequent flow of processes will lead the system to maximum entropy [3].

For the functioning of the biological system there are three possible cases:

- the level of organization of the system decreases;

- remains unchanged;

- grows.

At first sight it seems that the last two cases are related to the violations of the second law of thermodynamics. However, there is the development of living systems that are open, and the exchange of matter and energy with the environment takes place. In this case, the general change in the entropy ds in them occurs as a result of the development of irreversible processes with the release of heat $\delta Q_{i}$, as well as due to the external influx $\delta Q_{e}$ from the outside:

$$
d s=\frac{\delta Q_{e}}{T}+\frac{\delta Q_{i}}{T}=d_{e} s+d_{i} s
$$


For reverse processes $d_{i} s=0$, and for irreversible ones $d_{i} s>0$.

In an isolated system we also have $d_{e} s=0$, then:

$$
d_{i} s \geq 0
$$

By the differentiation of expression (3) we obtain:

$$
\frac{d s}{d t}=\frac{d_{e} s}{d t}+\frac{d_{i} s}{d t}
$$

Thus, the rate of change of the entropy of an open system is equal to the sum of the rates of entropy change due to interactions with the external environment and due to the change of entropy in the irreversible process of the system. Component $d_{i} s / d t>0$ because of its correspondence to an irreversible process, but the component $d_{e} s / d t$ may be less than zero. The overall result for the system is possible in three combinations:

$$
\begin{gathered}
\text { 1. } \frac{d s}{d t}>0 \text {,if } \frac{d_{e} s}{d t}>0 \text { and if } \frac{d_{e} s}{d t}<0 \text { and }\left|\frac{d_{e} s}{d t}\right|<\frac{d_{i} s}{d t} ; \\
\text { 2. } \frac{d s}{d t}<0 \text {, if } \frac{d_{e} s}{d t}<0 \text { and }\left|\frac{d_{e} s}{d t}\right|>\frac{d_{i} s}{d t} ; \\
\text { 3. } \frac{d s}{d t}=0 \text {, if } \frac{d_{e} s}{d t}<0 \text { and }\left|\frac{d_{e} s}{d t}\right|=\frac{d_{i} s}{d t} ;
\end{gathered}
$$

In the first case $(d s / d t>0)$, the degree of ordering of the system decreases. The second case corresponds to an increase in the order of order, and in the third case the stationary state of the system is achieved.

The above three cases are present in colloquia of carbon, water, oxygen and resorted to biotic and abiotic components. A special role is played by the process with decreasing entropy. Total energy metabolism of living organisms is associated with plant organisms that synthesize hydrocarbons from $\mathrm{H}_{2} \mathrm{O}$ and $\mathrm{CO}_{2}$. The oxidation of the latter in the processes of breathing of living organisms and microorganisms in the conditions of anaerobic and aerobic fermentation is accompanied by the release of energy, which ensures their livelihoods. Synthesis of hydrocarbons takes place in an environment of the atmosphere with the presence of elements of the lithosphere, the energy field of sunlight and the natural catalyst - grains of chlorophyll. At the same time, in the synthesized hydrocarbons, the energy potential is fixed in the form of chemical energy, and the Earth with its plant and biological world is a part of the system on a planetary scale. Thus, the implementation of systems with stabilized entropy or decreasing entropy requires an external or internal energy source.

Taking into account the "equality" of different types of energy sources, we turn to the estimation of culture environments of fermentation technologies. Their formation is grounded on the use of aqueous solutions of nutritional components (mainly - sugars) and 
yeast cultures. At the same time, the dissolution of sugars and yeast can be carried out at the level of self-destructive irreversible processes. In accordance with the meaning of the second law of thermodynamics, all real natural processes are irreversible, and the reciprocal processes are only their finite idealized cases [4]. Thus, the realization of any real process requires energy compensation for irreversible changes that arise in the system and in the culture environment.

The processes of fermentation with the aggregate of mass and energy exchange with the formation of $\mathrm{CO}_{2}$ and $\mathrm{C}_{2} \mathrm{H}_{5} \mathrm{OH}$ are also self-destructive and irreversible in the system "environment + microorganisms". The carrier of concentrated chemical energy is glucose, the oxidation of which liberates energy. The intermediate points of conservation of energy are chemical compounds in which electrons move to higher levels with energy consumption. When they return to previous energy levels, energy is released. The only natural compound - adenosinetriphosphate (ATP) stores a unit of energy and this unit of chemical energy can be converted into other forms of energy. It contains ATP of purine base (adenine), sugar with 5 carbon atoms (ribose) and three phosphoric acid residues. The connection between the external and the middle atom of phosphorus is particularly advantageous in energy terms. At its discontinuity there occurs to the emergence of adenosetidophosphate (ADP), phosphate, and there is an energy release of $30.5 \mathrm{~kJ}$ per mole of ATP:

$$
\mathrm{ATP} \rightarrow \mathrm{ADP}+\mathrm{P}+30.5 \mathrm{~kJ} / \mathrm{mol} .
$$

Approximately the same amount of energy is required for the transition from ADP to ATP, and ATP plays the role of an "energy storage" and a direct carrier of energy for all processes in cells that occur with energy expenditure or its release. At the same time, the transfer of energy is constantly and the accumulation of "energy bomb" becomes impossible. If the intake of glucose or other substances ceases, then there is no energy and the cell dies.

In yeast cells, the cytoplasm pyruvate synthesized in the mitochondria (cell power stations), where it is transformed into oxygen and water with a release of $38 \mathrm{ATP} /$ mole of glucose in the presence of oxygen, occurs. Therefore, in the presence of oxygen, yeast immediately pass to more energetically more desirable breathing.

The transformation of glucose into alcohol in the mode of anaerobic fermentation is accompanied by the difference in the free energy of Gibbs:

$$
\Delta \mathrm{G}=2870-2640=230 \mathrm{~kJ} / \text { mole } .
$$

For alcohol fermentation, chemical energy is stored in the form of two molecules of ATP and the cells consume only

$$
2 \cdot 30.5=61 \mathrm{~kJ} / \text { mole of glucose, }
$$

And the balance of $169 \mathrm{~kJ} / \mathrm{mol}$ of glucose is transformed into heat. Under the conditions of isobaric-isothermal process, the change in Gibbs energy is determined by dependence:

$$
\Delta \mathrm{G}=\Delta \mathrm{H}-\mathrm{T} \Delta \mathrm{S},
$$

where $\Delta \mathrm{H}-$ the change of the enthalpy of the medium. 


\section{— Processes and Equipment of Food Production -}

Thus, in the processes of anaerobic and aerobic fermentation, the Clausius theory is fully reflected with the direction of biochemical reactions and thermodynamic transformations in accordance with the principles of minimizing the energy potential of the system and the most probable state. The magnitude and velocity of Gibbs free energy directly determine the dynamics of the entropy change, as indicated by condition (12) and in the absence of forced stabilization the temperature of the culture medium would increase and eventually the fermentation process would stop with the subsequent lethal effect. So for the fermentation in each $\mathrm{m}^{3}$ of the environment $160 \mathrm{~kg}$ of glucose for the entire cycle of anaerobic process, the amount of heat energy that should stand out should be:

$$
Q_{i}=\frac{160 \cdot 169}{0,18}=150222,2 \mathrm{KJ}
$$

with an equivalent increase in temperature by

$$
\Delta t=\frac{Q_{i}}{c m}=\frac{150222,2}{4,19 \cdot 1000}=35,85^{\circ} \mathrm{C} .
$$

Under the condition that the nominal temperature of the medium is maintained at about $30 \ldots 32{ }^{\circ} \mathrm{C}$, compulsory cooling becomes obligatory. Aerobic fermentation is close to 1 million $\mathrm{kJ}$ per approximate to the above initial conditions.

If the consequent increase in entropy is due to the increase in temperature is simply enough neutralized by cooling the culture medium, the result of biochemical reactions in the form of synthesis of $\mathrm{C}_{2} \mathrm{~N}_{5} \mathrm{ON}$ and $\mathrm{CO}_{2}$ in modern anaerobic technologies with significant increase in the osmotic pressure is not subject to neutralization. Due to the absence of ethyl alcohol in the atmosphere, aerobic technology does not have such a disadvantage, and the increasing biological mass of microorganisms does not participate in the creation of osmotic pressure.

Returning to the effects of the processes of anaerobic fermentation, we emphasize that the saturation of the liquid phase with carbon dioxide in its quantitative characteristics is limited in accordance with Henry's law. However, although this limitation also applies to the osmotic pressure of dissolved $\mathrm{CO}_{2}$, there is another barrier to mass exchange on the surface of the phase separation "medium - microorganisms". The state of saturation of the liquid phase on $\mathrm{CO}_{2}$ complicates the release of cells from the synthesized gas in them, whereas the solubility of alcohol has no limitations. Reduction and stabilization of the concentrations of alcohol in environments is possible, for example, by the partial pressure reduction at reduced pressures, which uses the biological warming of the casting. In this case, the synthesized in $1 \mathrm{~m}^{3}$ of heat energy for the heat-vaporization of alcohol $\mathrm{kJ} / \mathrm{kg}$ should be sufficient to obtain $\mathrm{C}_{2} \mathrm{~N}_{5} \mathrm{ON}$ in the theoretical count in the amount:

$$
m_{c n}=\frac{Q_{i}}{r_{c n}}=164,9 \mathrm{~kg},
$$

which is almost twice the yield of alcohol.

The named energy-material relations lead to the conclusion about the possibility of a complete distillation process due to the transformed heat of fermentation using thermal transformers. 
The system of fermentation and distillation of wort in the production of ethanol (Figure 1) is provided to the use, it is shown in the scheme consisting of a crusher 1 grain, a mixer 2 grinding and water, a thermo-enzymatic treatment unit 3 , a solvent-refilling unit 4 , a pump 5 , a fermenter 6 with a cooling shirt 7 , which are connected to each other, the bragging column 8 , the cooling circuit 9 of the fermentation apparatus, the heat pump 10 in the composition of the evaporator 11 , the compressor 12 , the condenser 13 , the regulating valve 14 and the pumps 15 , the heating circuit 16 of the bragging column.

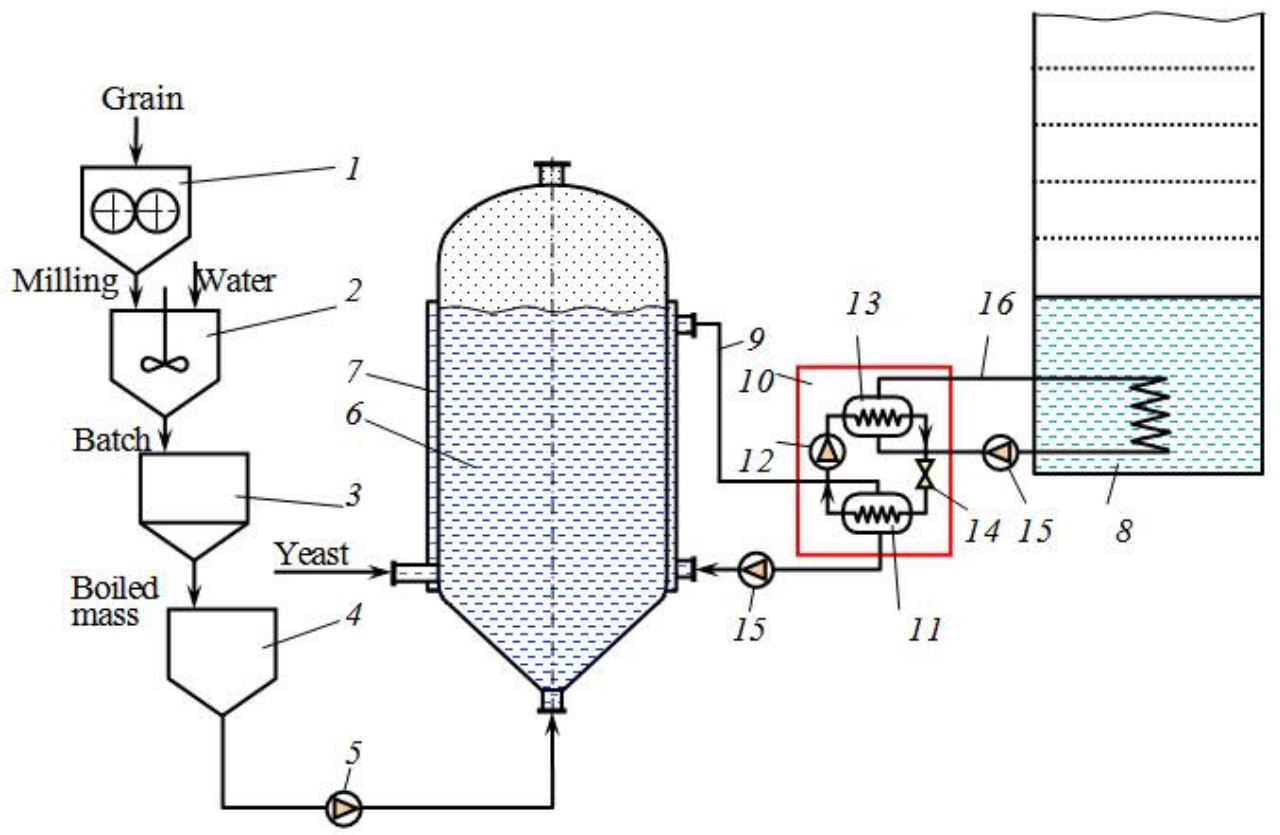

Figure 1. The scheme of the system of processing and distillation of the wort in the production of ethanol

The proposed system works in the following way. Recycled grain-weight mass is fed to a crusher, from which the milling enters the mixer, where the batch is prepared with water and enzymes. The latter is transmitted to the syrup, from which, after sacrifice, it enters the wart apparatus. The heat of digestion is diverted from the environment at the expense of the cooling shirt, stabilizing the temperature at the nominal value. The technical support of this process involves cooling the operating agent of the circuit 9 in the evaporator 11 of the heat pump.

The closed circuit 9 allows to create a cyclic process in which, in accordance with the 1st and 2nd laws of thermodynamics, there are processes of heating and cooling with the temperature-thermal circulation amplified by the pump 15. Under these conditions, a closed contour eliminates the loss of the working agent, which role can be performed by water. In addition, the thermodynamic deficiency of the covered heat exchange systems is reduced, which is associated with an increase in internal energy losses due to the final difference in the temperature of the heat carrier, and the possibility of adjusting the temperature 
fluctuations in the evaporator is achieved as a driving factor of transferring the heat. The evaporator formed in the vapor phase of the energy carrier of the heat pump is compressed by the compressor 12 with an increase in its temperature to $105 \ldots 110^{\circ} \mathrm{C}$ and transmitted to a condenser 13, where it condenses with the transfer of thermal current to the heating circuit of the turbine distillation column 16. Condensed heat-soak of the heat pump in the control valve is transformed with lowering the pressure and temperature and returns to the evaporator with subsequent transformations in the cycle. The heating circuit 16 of the medium of the refractory column ensures the formation of a steam in the alcohol mixture and the operation of the column in distillation. The result of such a transformation of the low-potential flow of heat of fermentation, which in modern schemes is accompanied by additional energy-consuming costs, is the complete recovery of secondary energy resources by utilizing the dual possibilities of heat pumps to obtain the source streams for the thermal stabilization of the cooling of fermented environments and for the heat-and-power supply of distillation processes. These advantages reveal the perspectives of the use of heat pumps in such systems, although the thermodynamic deficiencies include the presence of four heat transfer surfaces in the proposed system and the process of expanding the refrigerant in the throttle. At the same time there is a loss of pressure due to mechanical friction. Although there is no work in the process of expanding the working agent, and the throttle does not affect the amount of heat generated in the condenser, however, the amount of heat that is perceived in the evaporator at a constant temperature decreases. As a result, the interaction of the flow of the refrigerant with the throttle makes the process non-reversible and this feature is not excluded.

\section{Conclusions}

1. A quantitative indicator of the biological heat of fermentation is sufficient to ensure distillation of the parallel combination and execution of these processes.

2. The allocation of thermal energy in digestible media is the result of entropy losses of chemical energy in the form of free Gibbs energy.

3. The magnitude and rate of generating free energy of Gibbs in culture media is directly related to the dynamics of change in its entropy.

4. Technical support for the use of biological heat of fermentation for distillation processes is possible due to the use of thermal transformer grids in the form of closed energy circuits.

\section{References}

1. Shyan P. L. (1993), Development of resource and energy-saving technology and techniques of rectification in the food industry, USUFT, Kyiv.

2. Korolyuk K.E. (2001), Improvement of the technology of alcoholic beetles from starch-lagmiferous raw materials, USUFT, Kyiv.

3. Timanuk V.A., Zhivotova E.N. (2004), Biophysics, Professional, Kyiv.

4. Plank M. (1966), Unity of the Physical World View, Nauka, Moscow.

5. Kunze W. (2011), Technologie Brauer und Mälzer, LB Berlin, Berlin.

6. Natalia Tkachuk, Oleksandr Shevchenko (2016), Effects of osmotic pressure environments lethal effects on the level of microorganisms in the conditions of evacuation, Ukrainian Food Journal, 5(1), pp. 155-161. 
7. Thomson W., Tait P.G. (2009), Treatise on Natural Philosophy. Oxford. 2nd edition. Rissued by Cambridge University Press, Cambridge.

8. Monk Paul (2004), Physical Chemistry: Understanding our Chemical World, Wiley.

9. Tippens Paul E. (2007), Physics. 7th ed, McGraw-Hill.

10. Anderson T.M. (2009), Industrial Fermentation Processes, Encyclopedia of Microbiology (Third Edition), pp. 349-361

11. Atkins Peter, De Paula Julio (2006), Physical Chemistry (8th ed.), W.H. Freeman and Company.

12. Sokolenko A.I. (2011), Intensification of the heat and mass-transfer processes in the food technologies. Monography, Fenix, Kyiv.

13. Cooper Crystal (2010), Gay-Lussac's Law, Bright Hub Engineering, Available at: http://www.brighthubengineering.com/hvac/26213-gay-lussacs-law/

14. Shevchenko O.J. (2006), Scientific basis and hardware design of processes for long-term storage of food, NUFT, Kyiv.

15. Cropper William H. (2004), The Road to Entropy Rudolf Clausius, Great Physicists: The Life and Times of Leading Physicists from Galileo to Hawking, Oxford University Press, pp. 93-105.

16. Holtzer Alfred, Holtzer, Marilyn F. (2002), Use of the van't Hoff relation in determination of the enthalpy of micelle formation, The Journal of Physical Chemistry, 78(14), pp. 1442-1443.

17. Fernando Santos, Aluízio Borém and Celso Caldas (2015), Sugarcane. Agricultural Production, Bioenergy and Ethanol, Academic Press.

18. Stefan Hohmann (2002), Osmotic adaptation in yeast-control of the yeast osmolyte system, International Review of Cytology, 215, pp. 149-187

19. Stewart G.G., Priest F.G. (2011), Food and Beverage Stability and Shelf Life, Woodhead Publishing

20. Chahaida A.O., Piddubnyi V.A., Maksymenko I.F., Boiko O.O. (2013), Intensyfikatsiia zbrodzhuvannia tsukrovmistnykh seredovyshch, Scientific works of National University of Food Technologies, 51, pp. 77-83. 